\title{
Nonalcoholic Fatty Liver Disease: Dyslipidemia, Risk for Cardiovascular Complications, and Treatment Strategy
}

\author{
Qing-Qing Zhang and Lun-Gen Lu* \\ Department of Gastroenterology, Shanghai First People's Hospital, Shanghai Jiaotong University School of Medicine, Shanghai \\ 200080, China
}

\begin{abstract}
Studies have shown that nonalcoholic fatty liver disease (NAFLD) is strongly associated with several metabolic disorders and diseases, such as obesity, type 2 diabetes mellitus, and dyslipidemia. In NAFLD, dyslipidemia is manifested as increased serum triglyceride and low-density lipoprotein cholesterol levels and decreased high-density lipoprotein cholesterol levels, all of which are key risk factors for cardiovascular disease (CVD). CVD is a leading cause of mortality in NAFLD patients. Thus, implementation of an aggressive therapeutic strategy for dyslipidemia with hypolipidemic agents may mitigate the risk for CVD among NAFLD patients. Here, we provide a current review of literature regarding NAFLD, with particular emphasis on dyslipidemia and available treatment options.

(c) 2015 The Second Affiliated Hospital of Chongqing Medical University. Published by XIA \& HE Publishing Ltd. All rights reserved.
\end{abstract}

\section{Introduction}

Nonalcoholic fatty liver disease (NAFLD) is one of the most common chronic hepatic illnesses in developed countries, and its incidence among adults and adolescents has increased rapidly in recent years. In the United States, NAFLD represented over $75 \%$ of chronic liver disease cases in $2008,{ }^{1}$ a significant increase from 47\% estimated in 1998. Similarly, a high prevalence of NAFLD in the general population is found in Europe, where it is estimated to be $20-30 \% .^{2}$ NAFLD is associated with obesity, and between $30 \%$ and $100 \%$ of NAFLD patients are obese. ${ }^{3}$ The obese population is expanding at an alarming rate. For instance, a 2010

Keywords: Nonalcoholic fatty liver disease; Dyslipidemia; Cardiovascular disease; Hypolipidemic agents.

Abbreviations: $\mathrm{ApoB} / \mathrm{AI}$, apolipoprotein $\mathrm{B}$ /apolipoprotein $\mathrm{AI}$; $\mathrm{BMI}$, body mass index; CVD, cardiovascular disease; EASL, European Association for the Study of the Liver; FDA, Food and Drug Administration; FFA, free fatty acid; HDL-C, highdensity lipoprotein cholesterol; HMG-CoA, 3-hydroxy-3-methylglutaryl coenzyme A; LDL-C, low-density lipoprotein cholesterol; MS, metabolic syndrome; NAFLD, nonalcoholic fatty liver disease; NASH, nonalcoholic steatohepatitis; PPAR $\alpha$, peroxisome proliferator-activated receptor alpha; T2DM, type 2 diabetes mellitus; TG, high triglyceride; TLC, therapeutic lifestyle changes; TZDs, thiazolidinediones; VLDL, very low-density lipoprotein.

Received: 17 November 2014; Revised: 15 December 2014; Accepted: 22 December 2014

DOI: $10.14218 /$ JCTH.2014.00037.

*Correspondence to: Lun-Gen Lu, Department of Gastroenterology, Shanghai First People's Hospital, Shanghai Jiaotong University School of Medicine, Shanghai 200080, China. Tel: +86-21-63240090, Fax: +86-21-63241377, E-mail: lungenlu1965@ 163.com
Organization for Economic Cooperation and Development report indicated that approximately $15 \%$ of adults in Germany are obese (body mass index [BMI] $>30 \mathrm{~kg} / \mathrm{m}^{2}$ ) a clear increase from $2000(12 \%) .{ }^{4}$ By 2020, NAFLD is projected to become one of the most prevalent illnesses requiring a liver transplant. ${ }^{5,6}$

NAFLD refers to a liver that contains an excessive amount of fat, which is not related to alcohol consumption. Histologically, NAFLD consists of a wide spectrum of liver injury, including simple steatosis, nonalcoholic steatohepatitis (NASH), and NASH-related liver cirrhosis. Hepatocellular injury and subsequent inflammation in the liver tissue are characteristic pathological features of NASH. Among NASH patients, $15-20 \%$ will adversely progress to fibrosis, cirrhosis, or even carcinoma. ${ }^{7,8}$ The pathological hallmark of NAFLD is lipid accumulation in hepatocytes, suggesting there is a close link between abnormal lipid metabolism and NAFLD. Indeed, abnormal lipid metabolism due to other diseases can also lead to NAFLD.

The majority of NAFLD patients have metabolic syndrome (MS) as well. ${ }^{9}$ Thus, NAFLD is considered to be the hepatic manifestation of MS. ${ }^{10} \mathrm{~A}$ growing body of evidence supports an association between NAFLD and lipid MS, especially in type 7-9 NAFLD. ${ }^{11}$ In addition, the role of obesity, type 2 diabetes mellitus (T2DM), and dyslipidemia in the pathogenesis of NAFLD has been documented, ${ }^{12-14}$ implying that NAFLD may be an essential component of MS. Abnormal lipid metabolism or dyslipidemia in both MS-associated and non-MS-associated NAFLD can lead to cardiovascular complications (Fig. 1). Emerging evidence has shown the increased risk for coronary heart disease, left ventricular dysfunction, hypertrophy, heart failure, valvular heart disease, and arrhythmia in NAFLD patients. ${ }^{15}$ Therefore, both the liver and cardiovascular system should be closely managed in NAFLD. This review discusses dyslipidemia, the risk for cardiovascular complications, and the therapeutic strategy for NAFLD.

\section{Dyslipidemia and NAFLD}

Dyslipidemia that is characterized by high triglyceride (TG) and low high-density lipoprotein cholesterol (HDL-C) levels predisposes patients to arthrosclerosis. ${ }^{16}$ Approximately $20-80 \%$ of NAFLD patients also have dyslipidemia. ${ }^{17}$ A very common change in the metabolic profile among patients with T2DM, MS, and obesity is an alteration of serum lipid levels (dyslipidemia), suggesting a close relationship between T2DM, MS, and obesity and NAFLD. It has been shown that NASH significantly boosts the level of oxidized low-density lipoprotein cholesterol (LDL-C). High LDL-C is a well-established risk 


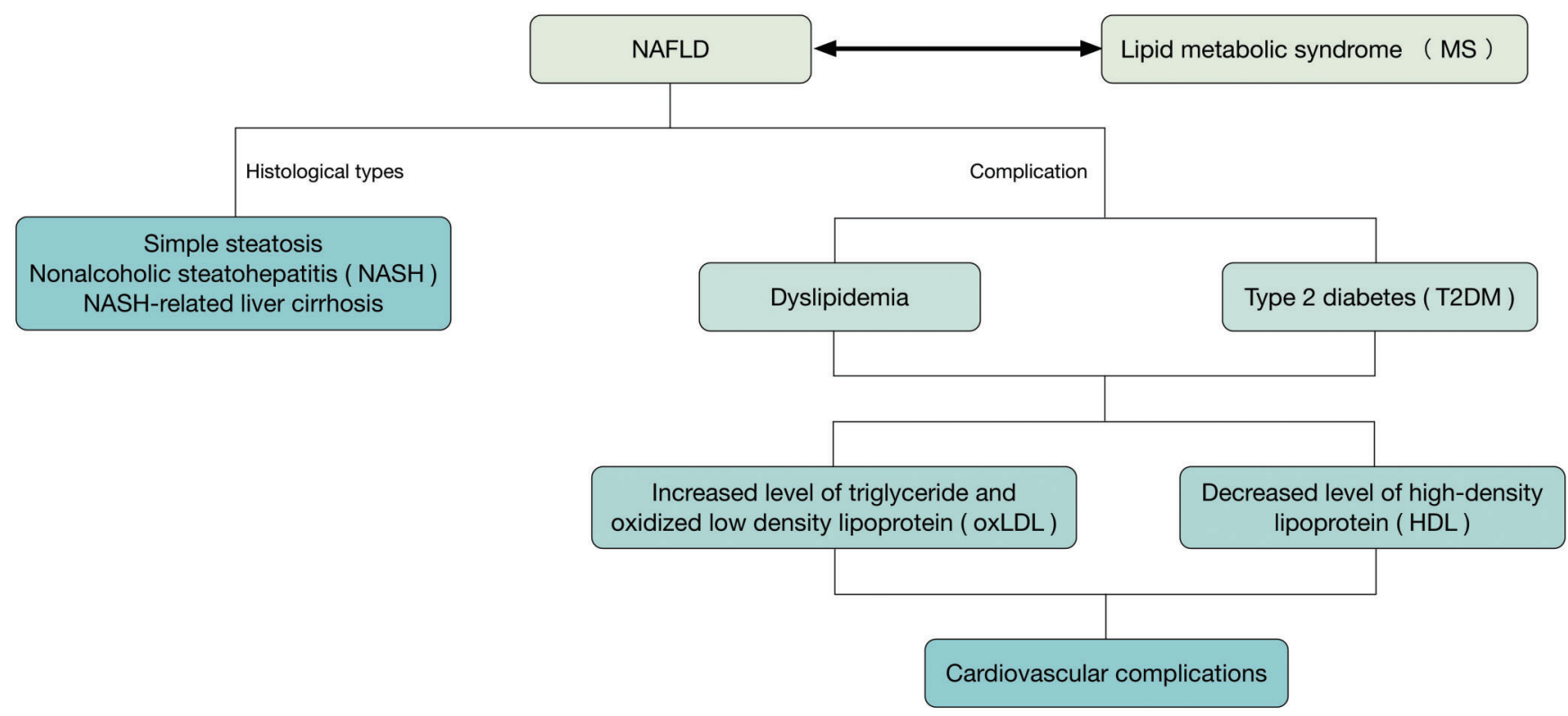

Fig. 1. The interrelationship among NAFLD, NASH, dyslipidemia, MS, and cardiovascular complications.

factor for arthrosclerosis. ${ }^{18}$ The most common form of dyslipidemia in NAFLD patients is atherogenic dyslipidemia, which is characterized by hypertriglyceridemia, low HDL-C levels, and high LDL-C levels. ${ }^{19}$ Long-term dyslipidemia may increase the expression and activity of sterol regulatory element binding protein-1c, a transcription factor, which adversely affects the profiles of lipid and lipoprotein synthesis in the liver, including increased TG, LDL, and very low-density lipoprotein (VLDL) levels and decreased HDL-C levels. ${ }^{20,21}$ There is a strong link between insulin resistance and metabolic dyslipidemia in T2DM. Insulin resistance facilitates the increase of free fatty acid (FFA) flux. The increased FFA level boosts TG and VLDL production as well as triggers oxidative stress and lipid peroxidation, all of which are closely associated with the development of NAFLD. ${ }^{22,23}$ Consequently, this physiological dysfunction also increases the risk for atherogenesis, thereby predisposing patients to cardiovascular diseases (CVDs). Direct evidence from a recent study supports a role of dyslipidemia in the development of steatosis. In an apolipoprotein E/low-density lipoprotein receptor doubleknockout mouse model with obvious dyslipidemia, a western diet adversely led to hepatic steatosis and fibrosis of the liver. ${ }^{24}$ Therefore, dysfunction of fat metabolism consequently leads to fat accumulation in the liver. In addition, circulating adipokines and cytokines as well as associated lipotoxicity, mitochondrial dysfunction, oxidative stress, and endoplasmic reticulum stress are involved in steatosis. ${ }^{25}$ It was recently demonstrated that the apolipoprotein B/apolipoprotein AI (ApoB/AI) ratio is a useful predictor of the risk of CV disease ${ }^{9,12}$ and is associated with the prevalence of NAFLD.

\section{CVDs and NAFLD}

Clinically, most NAFLD patients also have MS, thus predisposing these individuals to abnormal lipid metabolism in hepatocytes, leading to hepatocellular injury and inflammation. Therefore, NAFLD patients with MS exhibit a high tendency to develop NASH. ${ }^{8,26}$ Moreover, CVD risk factors, such as a high Framingham risk score, carotid intima media thickness, and high-sensitivity C-reactive protein level as well as early forms of atherosclerosis, endothelial dysfunction of the pericardial fat pads, and coronary artery calcification, are significantly elevated in NAFLD patients. ${ }^{27}$ Several longitudinal studies have demonstrated that cardiovascular events are the leading cause of mortality in NAFLD patients. ${ }^{28,29}$ In an eight year study, Matteoni et al. found that the mortality rate among 132 NAFLD patients was $36 \%$, and that most of them died of cardiovascular complications. ${ }^{30}$ The contribution of cardiovascular complications to the mortality of NAFLD patients was further confirmed by another clinical trial involving 132 NAFLD patients followed for 18 years. ${ }^{31}$ The aforementioned findings highlight the increased risk of developing secondary cardiovascular complications among NAFLD patients. However, the exact underlying mechanisms of this complex relationship remain not well understood. Some studies have suggested that inflammation, oxidative stress, insulin resistance, ectopic adipose tissue distribution, dyslipidemia, and endothelial dysfunction might be risk factors that link NAFLD and CVD. ${ }^{32}$ Regarding dyslipidemia as the plausible link between NAFLD and CVDs, aggressive management of dyslipidemia and prevention of cardiovascular deterioration may represent a promising addition to the treatment of NAFLD, thus reducing patient mortality. Liver fat deposition and oxidative stress result in the increased secretion of inflammatory markers, such as interleukin-6, tumor necrosis factor- $\alpha, C$-reactive protein, and fibrinogen. ${ }^{33}$ The inflammatory reaction boosts the expression of plasminogen activator inhibitor-1 and adhesion molecules in endothelial cells. Thus, the ideal therapeutic strategy for NAFLD management should not only reverse the excessive lipid accumulation in hepatocytes but also attenuate the hepatic inflammatory reaction, endothelial dysfunction, and insulin resistance in the liver, eventually preventing the progression of simple steatosis to NASH. A better understanding of the role of these factors in the pathogenesis of NAFLD is imperative for future studies. 
Zhang Q.Q. et al: Nonalcoholic fatty liver disease and dyslipidemia

\section{Management of dyslipidemia in NAFLD patients}

Management of abnormal lipid profiles is an important component of primary and secondary programs for preventing CVDs. Currently, a consensus on managing such lipid metabolic abnormalities in NAFLD patients, as adopted by the general guidelines, includes (a) individual patient assessment of lipid abnormalities; (b) stratification of patients according to the severity of the cardiovascular risk factors they have when admitted (high, medium, or low); (c) set target lipid levels for NAFLD; (d) aggressive lifestyle changes; and (e) hypolipidemic agents.

\section{Risk stratification}

The Canadian Cardiovascular Society has recommended risk stratification guidelines for the diagnosis and treatment of dyslipidemia and prevention of CVD. The stratification criteria utilize the Framingham risk score of patients who have concurrent diseases, such as T2DM, coronary artery disease, periphery vascular disease, and arthrosclerosis. ${ }^{34}$ Patients with a Framingham risk score greater than 20\%, 10-19\%, or less than $10 \%$ fall in the high-, medium-, and low-risk CVD risk categories, respectively.

\section{Therapeutic lifestyle changes}

A combination of diet and exercise, collectively known as therapeutic lifestyle changes (TLC), has been found to be critical for the overall health and wellness of participants. TLC is able to bring lipid levels within the acceptable range, ${ }^{35}$ which can significantly lower cardiovascular risk and favorably improve the clinical outcomes of NAFLD patients. ${ }^{36}$ The current recommendations for TLC include the following: a) eating food with less sugar, salt, and saturated fatty acids; b) maintaining a healthy body weight; c) exercising 30-60 min every day. The American College of Sports Medicine recommended in 2009 that moderate-intensity exercise between 150 and 250 min a week is effective in preventing weight gain and improves NAFLD. ${ }^{37}$ St George et al. ${ }^{38,39}$ showed that NAFLD patients who increased their physical activity by at least 60 min or who maintained their physical activity at $\geqslant 150$ min per week demonstrated the greater improvements in liver biochemistry compared to those who were inactive; d) quitting smoking; e) maintaining good mood and relieving pressure; and f) reducing alcohol consumption. In a one-year study, Huang et al. found that lifestyle changes and body weight loss resulted in significant histological improvement in patients with NASH ( $60 \%$ of 23 NAFLD patients). ${ }^{40}$ In another study, of the enrolled 49 dyslipidemic hemodialysis patients who completed a 21-week lifestyle intervention, $17 \%$ became nondyslipidemic and in other patients, the LDL cholesterol and triglyceride levels were significantly decreased and the HDL level was significantly increased. ${ }^{41}$

\section{Hypolipidemic agents and NAFLD}

Lipid-lowering compounds currently available are statins, fenofibrate, omega-3 fatty acids, niacin (table 1). These agents are widely used in the treatment of hyperlipidemia with monoor combination therapy, including patients with NAFLD. There are two major concerns with respect to the pharmacotherapeutic use clinically of these compounds for dyslipidemia in NAFLD. One is whether the current lipid-lowering compounds have the same efficacy for NAFLD as they have for other health issues accompanied with dyslipidemia. Another one is whether lipidlowering compounds can reduce the risk of CVD in patients with NAFLD.

\section{Statins}

Statins (or 3-hydroxy-3-methylglutaryl (HMG)-CoA reductase inhibitors) are a class of drugs that are used to lower cholesterol levels by blocking the enzyme HMG-CoA reductase. A number of statins are available on the market now: lovastatin, atorvastatin, fluvastatin, pitavastatin, pravastatin, rosuvastatin, and simvastatin. Statins inhibit the cholesterol synthesis pathway in the liver and significantly lower circulating cholesterol levels. Administration of statins induces a $20-60 \%$ reduction of LDL-C and TG levels while effectively increasing HDL-C levels by $1-10 \%$. Clinically, atorvastatin, pravastatin, simvastatin, and pitavastatin are garnering further attention because of their enhanced ability to lower hyperlipidemia in NAFLD patients.

\section{Atorvastatin}

Athyros et al. reported a clinical trial in Greece regarding the efficacy of atorvastatin on the prevention of secondary CVDs. ${ }^{42} \mathrm{~A}$ total of 1,600 subjects were enrolled in this three year follow-up study, of which 437 subjects were latent NAFLD patients without a definitive diagnosis. Among the latent NAFLD patients, 227 were administered atorvastatin ( $24 \mathrm{mg} /$ day) whereas 210 patients were not treated with a statin. They found that the patients treated with atorvastatin demonstrated a $35 \%, 44 \%$, and $32 \%$ reduction in the serum total cholesterol, LDL-C, and TG levels, respectively. Simultaneously, atorvastatin significantly increased the HDL-C level by $8 \%$ compared to the standard treatment group. Moreover, the cardiovascular risk in the atorvastatin group was significantly reduced by $68 \%$ (from $10 \%$ to $3.2 \%$ ). These findings suggest that statin treatment is safe and can improve liver tests and reduce cardiovascular morbidity in potential NAFLD patients with mild-to-moderately abnormal liver tests.

\section{Simvastatin}

Abel et al. reported that simvastatin ( $20 \mathrm{mg} /$ day) effectively improved the lipid profile of NAFLD patients in a six month nonblinded clinical trial. ${ }^{43}$ By using immunohistochemistry to measure LDL-C level in the liver, it was found that LDL-C in patients treated with simvastatin was reduced by $26 \%$ compared to untreated counterparts.

\section{Rosuvastatin}

In another prospective cohort study, 23 NAFLD patients were treated with rosuvastatin for 8 months. ${ }^{44}$ Lipid panel and liver function analyses were conducted prior to initiation of treatment and at 2 and 8 months during treatment. After 2 months, the patients showed a significant reduction of total cholesterol (33\%), LDL-C (41\%), TG (22\%), and transaminase $(50 \%)$ levels. Interestingly, all of these parameters were within the normal ranges. In addition, at 8 months of treatment, the HDL-C level was improved by $83 \%$ in NAFLD patients. 
Pravastatin

In a study with a small sample size, Rallidis et al. reported that pravastatin $(20 \mathrm{mg} /$ day) significantly affected total cholesterol level in five NAFLD patients. ${ }^{45}$ Although pravastatin treatment did not significantly impact the TG level, liver function in all patients was effectively improved after treatment. Moreover, notable amelioration of hepatic inflammation and steatosis was observed.

\section{Fibrates}

Fibrates are a class of amphipathic carboxylic acids, and they reduce lipid levels by activating peroxisome proliferatoractivated receptor alpha (PPAR $\alpha)$. PPARs are a class of intracellular receptors that modulate carbohydrate and fat metabolism as well as adipose tissue differentiation. PPAR $\alpha$ increases lipolysis and elimination of TG-rich particles from the plasma via activating lipoprotein lipase and reducing apolipoprotein CIII. ${ }^{46}$ Fibrates can improve the microcirculatory system function in the liver by facilitating fatty acid oxidation. It has been shown that gemfibrozil $(600 \mathrm{mg} /$ day) can significantly lower serum TG level and improve liver function of NAFLD patients. ${ }^{47}$ The results in this study are consistent with previous reports in which fenofibrate was found to be therapeutically beneficial on the lipid profile of NAFLD patients. ${ }^{48,49}$ In short, fibrates offer a different type of therapeutic strategy for the treatment of dyslipidemia in NAFLD patients.

\section{Ezetimibe}

Ezetimibe is a specific cholesterol absorption inhibitor, and it does not inhibit cholesterol synthesis in the liver or increase bile acid excretion. It lowers plasma cholesterol levels by decreasing cholesterol absorption in the small intestine. ${ }^{50}$ However, data regarding the overall efficacy data of ezetimibe are inconclusive for use in patients with NAFLD.

\section{Omega-3 fatty acids}

Omega-3 fatty acids are polyunsaturated fatty acids. They are essential fatty acids that cannot be endogenously synthesized. Omega-3 fatty acid monotherapy or in combination with a statin can lower the TG level without affecting the LDL-C level. ${ }^{51}$ Omega- 3 fatty acids have been approved by the United States Food and Drug Administration (FDA, US) as an adjunct to diet for lowering very high TG levels. ${ }^{52}$ Previous studies have found that omega-3 fatty acids can effectively improve lipid profiles in NAFLD patients treated for eight to 12 months. ${ }^{53-57}$ Although results from studies examining liver function tests after treatment with omega-3 fatty acids are inconsistent, all studies have strong clinical evidence in support of the benefits of omega-3 fatty acids on TG level in NAFLD patients. For example, in a clinical trial, 144 NAFLD patients were administered 29 omega-3 fatty acids or placebo daily for 24 weeks. Aminotransferase and serum TG levels as well as hepatic steatosis were significantly improved in the patients administered the omega- 3 fatty acids compared to the placebo group.

\section{Combination Therapies}

To achieve a maximal reduction in the LDL-C level, the combination of statins with other lipid-lowering agents has been evaluated. In a study by Athyros et al., 186 NAFLD patients were treated with atorvastatin $(20 \mathrm{mg} /$ day $)$, fenofibrate $(200 \mathrm{mg} / \mathrm{day})$, or a combination of these for 54 weeks; and it was found that outcomes on lowering cardiovascular risk were better with atorvastatin than fenofibrate. ${ }^{48}$ Interestingly, the combination treatment was also more efficacious than fenofibrate alone. In another similar study, Foster et al. found that the combination of atorvastatin (20 mg/day) with vitamin C (1 g/day or $1000 \mathrm{IU} /$ day) greatly improved the survival of NAFLD patients at the end of the follow-up period. ${ }^{58}$ Moreover, their data suggested that the beneficial effects on hepatic steatosis were closely associated with a decrease in the total cholesterol and LDL-C levels but not HDL-C or TG levels.

\section{Statins and the risk of serious hepatotoxicity}

Although up to $2 \%$ of the patients treated with statins show elevated alanine aminotransferase levels at more than three times the upper limit of normal, the risk of serious hepatotoxicity is still very rare. However, the significant increase of aminotransferase levels in some treated patients does raise concerns regarding the potential hepatotoxicity of statins. Recently, numerous studies have demonstrated that statins are safe for treating NAFLD patients. ${ }^{59-62}$ There is no clinical evidence that hyperlipidemic patients with high aminotransferase levels possess a greater risk for statin hepatotoxicity than their counterparts with normal transaminase levels. A clinical study by Chalasani et al. clearly confirmed this conclusion. ${ }^{60}$ In this study, 342 hyperlipidemic patients with high aminotransferase levels (cohort 1) and 1,437 hyperlipidemic patients with normal aminotransferase levels (cohort 2 ) received statin therapy. An additional 2,245 patients with elevated liver enzyme levels (cohort 3) who did not receive statins (liver disease control) were also enrolled in this study. After 6 months of treatment, hepatotoxity of statins was examined. The incidence of mild-moderate elevations in liver biochemistries in cohort 2 was less than cohort 1 (1.9\%, $p=0.002$ ), but there was no significant difference between cohort 2 and cohort 1 in severe elevations $(0.2 \%, p=0.2)$. There were no differences in liver biochemistries between cohorts 1 and 3 . In another clinical trial that was designed to examine the safety of pravastatin, it was found that the incidence of elevated aminotransferase levels was not significantly different between pravastatin-treated and placebo-treated patients. ${ }^{52}$ Overall, the available data suggest that statin-induced hepatotoxicity is quite rare.

\section{Insulin sensitizing agents and NAFLD}

Metformin, an inhibitor of hepatic gluconeogenesis, is used clinically as a first-line treatment of T2DM. In an open label trial consisting of 110 patients randomly assigned to receive either metformin $2 \mathrm{~g} / \mathrm{d}$ (55 patients), vitamin E $800 \mathrm{IU} / \mathrm{d}$ (28 patients), or dietary-induced weight loss (27 patients) patients for 12 months, liver transaminase levels were significantly decreased in the metformin group relative to the other groups. Also, in a subset of 17 patients taking metformin, there were histological improvements in hepatic steatosis, inflammation, and fibrosis. A recent clinical trial, however, did not find any significant benefit of treatment in pediatric patients with the disease. ${ }^{63}$ Meanwhile, the position statement based on an European Association for the Study of the Liver (EASL) special conference on NAFLD/NASH has not 
Zhang Q.Q. et al: Nonalcoholic fatty liver disease and dyslipidemia

Table 1. Overview of dyslipidemia agents in NAFLD

\begin{tabular}{|c|c|c|c|}
\hline Dyslipidemia agents & Brief description & Representative drugs & Effects on the liver \\
\hline Statins & $\begin{array}{l}\text { A class of drugs that are used to } \\
\text { lower cholesterol levels by block- } \\
\text { ing the enzyme HMG-CoA reduc- } \\
\text { tase }\end{array}$ & $\begin{array}{l}\text { Atorvastatin } \\
\text { Simvastatin } \\
\text { Rosuvastatin } \\
\text { Pravastatin }\end{array}$ & $\begin{array}{l}\text { Inhibit the cholesterol synthesis } \\
\text { pathway in the liver and signifi- } \\
\text { cantly lower circulating cholesterol } \\
\text { levels by reducing endogenous } \\
\text { cholesterol synthesis. }\end{array}$ \\
\hline Fibrates & $\begin{array}{l}\text { A class of amphipathic carboxylic } \\
\text { acids used to reduce lipid levels } \\
\text { by activating peroxisome prolif- } \\
\text { erator-activated receptor alpha } \\
(\text { PPAR } \alpha)\end{array}$ & Fibrates & $\begin{array}{l}\text { Improve serum liver enzymes } \\
\text { without affecting liver histology } \\
\text { and improve the microcirculatory } \\
\text { system function in the liver by } \\
\text { facilitating fatty acid oxidation. }\end{array}$ \\
\hline Ezetimibe & $\begin{array}{l}\text { A specific cholesterol absorption } \\
\text { inhibitor }\end{array}$ & Ezetimibe & $\begin{array}{l}\text { Not tested in RCTs. Lower plasma } \\
\text { cholesterol levels by decreasing } \\
\text { cholesterol absorption in the small } \\
\text { intestine. The overall efficacy data } \\
\text { are inconclusive in NAFLD. }\end{array}$ \\
\hline $\begin{array}{l}\text { Omega-3 fatty } \\
\text { acids }\end{array}$ & Polyunsaturated fatty acids & Omega-3 fatty acids & $\begin{array}{l}\text { Lower the TG level without affect- } \\
\text { ing the LDL-C level in NAFLD. }\end{array}$ \\
\hline $\begin{array}{l}\text { Insulin sensitizing } \\
\text { agents }\end{array}$ & $\begin{array}{l}\text { Activates AMPK and improves } \\
\text { peripheral insulin sensitivity }\end{array}$ & $\begin{array}{l}\text { Metformin } \\
\text { Thiazolidinediones } \\
\text { (TZDs) }\end{array}$ & $\begin{array}{l}\text { Improve liver histology, glucose, } \\
\text { lipid, and inflammatory variables } \\
\text { and delay progression to fibrosis } \\
\text { in patients with NAFLD }\end{array}$ \\
\hline
\end{tabular}

recommended metformin for specific liver-directed therapy of $\mathrm{NASH}{ }^{64}$

The thiazolidinediones (TZDs) are another group of insulin sensitizing agents that improve insulin sensitivity at the level of adipose tissue, muscle, and liver and are now widely used for treatment of T2DM. Troglitazone was the first TZD to be tested for NASH in an open-label pilot clinical trial. ${ }^{65} \mathrm{~A}$ recent meta-analysis showed the beneficial effects of pioglitazone on liver histology, glucose, lipid, and inflammatory variables, and progression to fibrosis in patients with NAFLD. ${ }^{66}$ The drug should be considered for treatment of NAFLD, especially in patients with T2DM.

\section{Conclusions}

NAFLD patients frequently have MS, especially dyslipidemia. The features of dyslipidemia include elevated TG and LDL-C levels accompanied with a decreased HDL-C level. NAFLD patients carry a high risk for CVDs, which are a leading cause of mortality for NAFLD patients; therefore, reducing the CVD risk could represent a pivotal strategy for effectively managing NAFLD patients. In addition, correction of abnormal lipid profiles in these patients could improve their survival by lowering the risk of CVDs. The criteria for CVD risk stratification of NAFLD patients have been established, and the corresponding management should be based on the risk status of the patients. Statins are the first line choice for treating hypercholesterolemia. The drug dosage should be adjusted individually based on tolerability and the therapeutic target. As reviewed here, all statins seem to restore the altered serum lipid levels in NAFLD patients effectively. However, physicians may prefer atorvastatin to other drugs, since atorvastatin is the only agent that has been proven to lower cardiovascular risk factors in NAFLD patients and possesses less hepatotoxicity. For the treatment of hypertriglyceridemia, omega- 3 fatty acids may be the first choice, because of their enhanced ability to lowering serum TG levels and they are well tolerated. Importantly, current evidence suggests that combination therapy with more than a hypolipidemic agent in NAFLD patients may increase efficacy in controlling dyslipidemia and improve tolerability.

\section{Conflict of interest}

None

\section{Author contributions}

Designing the research (ZQQ, LLG), searching the databases and writing the paper (ZQQ), making the complete guide of article (LLG).

\section{References}

[1] Younossi ZM, Stepanova M, Afendy M, Fang Y, Younossi $Y$, Mir $H$, et al. Changes in the prevalence of the most common causes of chronic liver diseases in the United States from 1988 to 2008. Clin Gastroenterol Hepatol 2011;9:524-530. doi: 10.1016/j.cgh.2011.03.020.

[2] Blachier M, Leleu H, Peck-Radosavljevic M, Valla DC, Roudot-Thoraval F. The burden of liver disease in Europe. A Review of available epidemiological data. J Hepatol 2013;58:593-608. doi: 10.1016/j.jhep.2012.12.005.

[3] Angulo P. Nonalcoholic fatty liver disease. N Engl J Med 2002;346:2121-1231.

[4] http://dx.doi.org/10.1787/9789264183896-en, accessed April 2014.

[5] Williams CD, Stengel J, Asike MI, Torres DM, Shaw J, Contreras M, et al. Prevalence of nonalcoholic fatty liver disease and nonalcoholic steatohepatitis among a largely middle-aged population utilizing ultrasound and liver biopsy: a prospective study. Gastroenterology 2011;140:124-131. doi: 10.1053/j.gastro.2010.09.038.

[6] Charlton MR, Burns JM, Pedersen RA, Watt KD, Heimbach JK, Dierkhising RA. Frequency and outcomes of liver transplantation for nonalcoholic steatohepatitis in the United States. Gastroenterology 2011;141:1249-1253. doi: 10.1053/j.gastro.2011.06.061.

[7] Scott C. Matherly, Puneet Puri. Mechanisms of Simple Hepatic Steatosis: Not So Simple After All. Clin Liver Dis 2012;16:505-524. doi: 10.1016/j.cld.2012.05.005.

[8] Vuppalanchi R, Chalasani N. Nonalcoholic fatty liver disease and nonalcoholic steatohepatitis: selected practical issues in their evaluation and management. Hepatology 2009;49:306-317. doi: 10.1002/hep.22603. 
[9] Dima A, Marinescu AG, Dima AC. Non-alcoholic fatty liver disease and the statins treatment. Rom J Intern Med 2012, 50:19-25.

[10] Ji C, Dai Y, Jiang W, Liu J, Hou M, Wang J, et al. Postnatal overfeeding promotes early onset and exaggeration of high-fat diet-induced nonalcoholic fatty liver disease through disordered hepatic lipid metabolism in rats. J Nutr Biochem 2014;25: 1108-1116. doi: 10.1016/j.jnutbio.2014.06.010.

[11] Neuschwander-Tetri BA, Clark JM, Bass NM, Van Natta ML, Unalp-Arida A, Tonascia J, et al. NASH Clinical Research Network. Clinical, laboratory and histological associations in adults with nonalcoholic fatty liver disease. Hepatology 2010;52:913-924. doi: 10.1002/hep.23784.

[12] Kalra S, Vithalani M, Gulati G, Kulkarni CM, Kadam Y, Pallivathukkal J, et al. Study of prevalence of nonalcoholic fatty liver disease (NAFLD) in type 2 diabetes patients in India (SPRINT). J Assoc Physicians India 2013;61:448453.

[13] Boyraz M, Hatipoğlu N, Sarı E, Akcay A, Taskin N, Ulucan K, et al. Nonalcoholic fatty liver disease in obese children and the relationship between metabolic syndrome criteria. Obes Res Clin Pract 2014;8:e356-e363. doi: 10.1016/j.orcp.2013.08.003.

[14] Sahebkar A, Chew GT, Watts GF. New peroxisome proliferator-activated receptor agonists: potential treatments for atherogenic dyslipidemia and non-alcoholic fatty liver disease. Expert Opin Pharmacother 2014;15:493503. doi: 10.1517/14656566.2014.876992.

[15] Ballestri S, Lonardo A, Bonapace S, Byrne CD, Loria P, Targher G. Risk of cardiovascular, cardiac and arrhythmic complications in patients with nonalcoholic fatty liver disease. World J Gastroenterol 2014;20:1724-1745. doi: 10.3748/wjg.v20.i7.1724.

[16] Targher G, Day CP, Bonora E. Risk of cardiovascular disease in patients with nonalcoholic fatty liver disease. N Engl J Med 2010;363:1341-1350. doi: 10.1056/NEJMra0912063.

[17] Souza MR, Diniz MF, Medeiros JE, Araujo MS. Metabolic syndrome and risk factors for non-alcoholic fatty liver disease. Arq Gastroenterol 2012;49:8996.

[18] Norris AL, Steinberger J, Steffen LM, Metzig AM, Schwarzenberg SJ, Kelly AS. Circulating oxidized LDL and inflammation in extreme pediatric obesity. Obesity 2011;19:1415-1419. doi: 10.1038/oby.2011.21.

[19] Sahebkar A1, Chew GT, Watts GF. New peroxisome proliferator-activated receptor agonists: potential treatments for atherogenic dyslipidemia and non-alcoholic fatty liver disease. Expert Opin Pharmacother 2014, 15:493503. doi: 10.1517/14656566.2014.876992.

[20] Ahmed MH, Abu EO, Byrne CD. Non-Alcoholic Fatty Liver Disease (NAFLD): new challenge for general practitioners and important burden for health authorities? Prim Care Diabetes 2010;4:129-137. doi: 10.1016/j.pcd.2010. 02.004 .

[21] Gawrieh S, Baye TM, Carless M, Wallace J, Komorowski R, Kleiner DE, et al. Hepatic gene networks in morbidly obese patients with nonalcoholic fatty liver disease. Obes Surg 2010;20:1698-1709. doi: 10.1007/s11695-0100171-6.

[22] Alkhouri N, Eng K, Lopez R, Nobili V. Non-high-density lipoprotein cholesterol (non-HDL-C) levels in children with nonalcoholic fatty liver disease (NAFLD). Springerplus 2014;3:407. doi: 10.1186/2193-1801-3-407.

[23] Cao W, Zhao C, Shen C, Wang Y. Cytokeratin 18, alanine aminotransferase, platelets and triglycerides predict the presence of nonalcoholic steatohepatitis. PLOS One 2013;8:e82092. doi: 10.1371/journal.pone.0082092.

[24] Kampschulte M, Stockl C, Langheinrich AC, Althohn U, Bohle RM, Krombach $\mathrm{GA}$, et al. Western diet in ApoE-LDLR double-deficient mouse model of atherosclerosis leads to hepatic steatosis, fibrosis, and tumorigenesis. Lab Invest 2014;94:1273-1282. doi: 10.1038/labinvest.2014.112.

[25] Koppe SW. Obesity and the liver: nonalcoholic fatty liver disease. Transl Res 2014, 164:312-322. doi: 10.1016/j.trsl.2014.06.008.

[26] Angulo P. Nonalcoholic fatty liver disease. N Engl J Med 2002;346:12211231.

[27] Speliotes EK, Massaro JM, Hoffmann U, Vasan RS, Meigs JB, Sahani DV, et al. Fatty liver is associated with dyslipidemia and dysglycemia independent of visceral fat: the Framingham Heart Study. Hepatology 2010;51:1979-1987. doi: $10.1002 /$ hep. 23593.

[28] Söderberg C, Stảl P, Askling J, Glaumann H, Lindberg G, Marmur J, et al. Decreased survival of subjects with elevated liver function tests during a 28 year follow-up. Hepatology 2010;51:595-602. doi: 10.1002/hep.23314.

[29] Ekstedt M, Franzén LE, Mathiesen UL, Thorelius L, Holmqvist M, Bodemar G, et al. Long-term follow-up of patients with NAFLD and elevated liver enzymes. Hepatology 2006;44:865-873. doi: 10.1002/hep.21327.

[30] Matteoni CA, Younossi ZM, Gramlich T, Boparai N, Liu YC, McCullough AJ. Nonalcoholic fatty liver disease: a spectrum of clinical and pathological severity. Gastroenterology 1999;116:1413-1419. doi: 10.1016/S00165085(99)70506-8.

[31] Rafiq N, Bai C, Fang Y, Srishord M, McCullough A, Gramlich T, et al. Long-term follow-up of patients with nonalcoholic fatty liver. Clin Gastroenterol Hepatol 2009;7:234-238. doi: 10.1016/j.cgh.2008.11.005.

[32] Liu H, Lu HY. Nonalcoholic fatty liver disease and cardiovascular disease. World J Gastroenterol 2014;20:8407-8415. doi: 10.3748/wjg.v20.i26.8407.
[33] Gaggini M, Morelli M, Buzzigoli E, DeFronzo RA, Bugianesi E, Gastaldelli A. Non-alcoholic fatty liver disease (NAFLD) and its connection with insulin resistance, dyslipidemia, atherosclerosis and coronary heart disease. Nutrients 2013;5:1544-1560. doi: 10.3390/nu5051544.

[34] Genest J, McPherson R, Frohlich J, Anderson T, Campbell N, Carpentier A, et al. Canadian Cardiovascular Society/Canadian guidelines for the diagnosis and treatment of dyslipidemia and prevention of cardiovascular disease in the adult-2009 recommendations. Can J Cardiol 2009;25:567-579.

[35] Managing your dyslipidemia with TLC: Therapeutic Lifestyle Changes. J Am Osteopath Assoc 2010, 110:eS17-eS18.

[36] Lam B, Younossi ZM. Treatment options for nonalcoholic fatty liver disease. Therap Adv Gastroenterol 2010;3:121-137. doi: 10.1177/1756283X09359964.

[37] Fock KM, Khoo J. Diet and exercise in management of obesity and overweight. J Gastroenterol Hepatol 2013;28(Suppl):59-63. doi: 10.1111/jgh.12407.

[38] St George A, Bauman A, Johnston A, Farrell G, Chey T, Georgr J. The independent effects of physical activity in patients with non-alcoholic fatty liver disease. Hepatology 2009;50:68-76. doi: 10.1002/hep.22940.

[39] St George A, Bauman A, Johnston A, Farrell G, Chey T, George J. Effect of lifestyle intervention in patients with abnormal liver enzymes and metabolic risk factors. J Gastroenterol Hepatol 2009;24:399-407. doi: 10.1111/ j.1440-1746.2008.05694.x

[40] Huang MA, Greenson JK, Chao C, Anderson L, Peterman D, Jacobson J, et al. One-year intense nutritional counseling results in histological improvement in patients with nonalcoholic steatohepatitis: a pilot study. Am J Gastroenterol 2005;100:1072-1081. doi: 10.1111/j.1572-0241.2005.41334.x.

[41] Grzegorzewska AE, Niepolski L, Sikora J, Jankow M, Jagodzinski PP, Sowinska A. Effect of lifestyle changes and atorvastatin administration on dyslipidemia in hemodialysis patients: a prospective study. Pol Arch Med Wewn 2014;124: 443-451.

[42] Athyros VG, Tziomalos K, Gossios TD, Griva T, Anagnostis P, Kargiotis K, et al. Safety and efficacy of long-term statin treatment for cardiovascular events in patients with coronary heart disease and abnormal liver tests in the Greek Atorvastatin and Coronary Heart Disease Evaluation (GREACE) Study: a post-hoc analysis. Lancet 2010;376:1916-1922. doi: 10.1016/S01406736(10)61272-X.

[43] Nelson A, Torres DM, Morgan AE, Fincke C, Harrison SA. A pilot study using simvastatin in the treatment of nonalcoholic steatohepatitis: A randomized placebo-controlled trial. J Clin Gastroenterol 2009;43:990-994. doi: 10.1097/MCG.0b013e31819c392e.

[44] Antonopoulos S, Mikros S, Mylonopoulou M, Kokkoris S, Giannoulis G. Rosuvastatin as a novel treatment of non-alcoholic fatty liver disease in hyperlipidemic patients. Atherosclerosis 2006;184:233-234. doi: 10.1016/ j.atherosclerosis.2005.08.021.

[45] Rallidis LS, Drakoulis CK, Parasi AS. Pravastatin in patients with nonalcoholic steatohepatitis: results of a pilot study. Atherosclerosis 2004;174:193-196. doi: 10.1016/j.atherosclerosis.2004.01.008

[46] Staels B, Dallongeville J, Auwerx J, Schoonjans K, Leitersdorf E, Fruchart JC. Mechanism of action of fibrates on lipid and lipoprotein metabolism. Circulation 1998;98:2088-2093. doi: 10.1161/01.CIR.98.19.2088.

[47] Basaranoglu M, Acbay O, Sonsuz A. A controlled trial of gemfibrozil in the treatment of patients with nonalcoholic steatohepatitis. J Hepatol 1999;31: 384. doi: 10.1016/S0168-8278(99)80243-8.

[48] Athyros VG, Mikhailidis DP, Didangelos TP, Giouleme OI, Liberopoulos EN, Karagiannis $A$, et al. Effect of multifactorial treatment on non-alcoholic fatty liver disease in metabolic syndrome: a randomized study. Curr Med Res Opin 2006;22:873-883. doi: 10.1185/030079906X104696.

[49] Fernández-Miranda C, Pérez-Carreras M, Colina F, Lopez-Alonso G, Vargas C, Solis-Herruzo JA. A pilot trial of fenofibrate for the treatment of non-alcoholic fatty liver disease. Dig Liver Dis 2008;40:200-205. doi: 10.1016/j.dld.2007. 10.002.

[50] Davidson HM. Ezetimibe: a novel option for lowering cholesterol. Expert Rev Cardiovasc Ther 2003;1:11-21. doi: $10.1586 / 14779072.1 .1 .11$.

[51] Weintraub $\mathrm{H}$. Update on marine omega-3 fatty acids: management of dyslipidemia and current omega-3 treatment options. Atherosclerosis 2013 230:381-389. doi: 10.1016/j.atherosclerosis.2013.07.041

[52] McKenney JM, Sica D. Role of prescription omega-3 fatty acids in the treatment of hypertriglyceridemia. Pharmacotherapy 2007;27:715-728. doi: 10.1592/phco.27.5.715.

[53] Capanni M, Calella F, Biagini MR, Genise S, Raimondi L, Bedogni G, et al. Prolonged omega- 3 polyunsaturated fatty acid supplementation ameliorates hepatic steatosis in patients with non-alcoholic fatty liver disease: a pilot study. Aliment Pharmacol Ther 2006;23:1143-1151. doi: 10.1111/j.13652036.2006.02885.x.

[54] Spadaro L, Magliocco O, Spampinato D, Piro S, Oliveri C, Alagona C, et al. Effects of omega-3 polyunsaturated fatty acids in subjects with nonalcoholic fatty liver disease. Dig Liver Dis 2008;40:194-199. doi: 10.1016/j.dld.2007.10.003.

[55] Tanaka N, Sano K, Horiuchi A, Tanaka E, Kiyosawa K, Aoyama T. Highly purified eicosapentaenoic acid treatment improves nonalcoholic steatohepatitis. J Clin Gastroenterol 2008;42:413-418. doi: 10.1097/MCG.0b013 e31815591aa. 
[56] Vega GL, Chandalia M, Szczepaniak LS, Grundy SM. Effects of N-3 fatty acids on hepatic triglyceride content in humans. J Investig Med 2008;56:780-785. doi: 10.231/JIM.0b013e318177024d.

[57] Itoh M, Suganami T, Satoh N, Tanimoto-Koyama K, Yuan X, Tanaka M, et al. Increased adiponectin secretion by highly purified eicosapentaenoic acid in rodent models of obesity and human obese subjects. Arterioscler Thromb Vasc Biol 2007;27:1918-1925. doi: 10.1161/ATVBAHA.106.136853.

[58] Foster T, Budoff MJ, Saab S, Ahmadi N, Gordon C, Guerci AD. Atorvastatin and antioxidants for the treatment of nonalcoholic fatty liver disease: the St Francis Heart Study randomized clinical trial. Am J Gastroenterol 2011;106: 71-77. doi: 10.1038/ajg.2010.299.

[59] Lewis JH, Mortensen ME, Zweig S, Fusco MJ, Medoff JR, Belder R. Efficacy and safety of high-dose pravastatin in hypercholesterolemic patients with wellcompensated chronic liver disease: Results of a prospective, randomized, double-blind, placebo-controlled, multicenter trial. Hepatology 2007;46: 1453-1463. doi: 10.1002/hep.21848.

[60] Chalasani N, Aljadhey H, Kesterson J, Murray MD, Hall SD. Patients with elevated liver enzymes are not at higher risk for statin hepatotoxicity. Gastroenterology 2004;126:1287-1292. doi: 10.1053/j.gastro.2004. 02.015.

[61] Pfeffer MA, Keech A, Sacks FM, Cobbe SM, Tonkin A, Byington RP, et al. Safety and tolerability of pravastatin in long-term clinical trials: prospective
Pravastatin Pooling (PPP) Project. Circulation 2002;105:2341-2346. doi: 10.1161/01.CIR.0000017634.00171.24.

[62] Mateen FJ, Kanters S, Kalyesubula R, Mukasa B, Kawuma E, Kengne AP, et al. Hypertension prevalence and Framingham risk score stratification in a large HIV-positive cohort in Uganda. J Hypertens 2013;31:1372-1378. doi: 10.1097/HJH.0b013e328360de1c.

[63] Lavine JE, Schwimmer JB, Natta ML, Molleston JP, Murray KF, Rosenthal P. Effect of vitamin $E$ or metformin for treatment of nonalcoholic fatty liver disease in children and adolescents: the TONIC randomized controlled trial. JAMA 2011;305:1659-1668. doi: 10.1001/jama.2011.520.

[64] Ratziu V, Bellentani S, Cortez-Pinto H, Day C, Marchesini G. A position statement on NAFLD/NASH based on the EASL 2009 special conference. J Hepatol 2010;53:372-384. doi: 10.1016/j.jhep.2010.04.008.

[65] Caldwell SH, Hespenheide EE, Redick JA, Iezzoni JC, Battle EH, Sheppard BL. A pilot study of a thiazolidinedione, troglitazone, in nonalcoholic steatohepatitis. Am J Gastroenterol 2001;96:519-525. doi: 10.1111/j.15720241.2001.03553.x

[66] Musso G, Cassader G, Rosina F, Gambino F. Impact of current treatments on liver disease, glucose metabolism and cardiovascular risk in non-alcoholic fatty liver disease (NAFLD): a systematic review and meta-analysis of randomised trials. Diabetologia 2012;55:885-904. doi: 10.1007/s00125011-2446-4. 